\title{
Downregulation of USP12 inhibits tumor growth via the p38/MAPK pathway in hepatocellular carcinoma
}

\author{
CHUNSHENG LIU ${ }^{1 *}$, XIAONING LI ${ }^{1 *}$, GANG FENG $^{1}$, MINGGANG CAO $^{2}$, \\ FAHU LIU ${ }^{1}$, GUIHUA ZHANG ${ }^{1}$ and YONG LU ${ }^{3 *}$ \\ ${ }^{1}$ Department of Laboratory Medicine, Yijishan Hospital of Wannan Medical College, Wuhu, Anhui 241002; \\ ${ }^{2}$ Department of Medicine, Anhui College of Traditional Chinese Medicine, Wuhu, Anhui 241000; \\ ${ }^{3}$ School of Laboratory Medicine, Wannan Medical College, Wuhu, Anhui 241002, P.R. China
}

Received May 26, 2019; Accepted January 23, 2020

DOI: $10.3892 / \mathrm{mmr} .2020 .11557$

\begin{abstract}
Hepatocellular carcinoma (HCC) is one of the most common malignancies worldwide. Ubiquitin-specific protease 12 (USP12) is specifically upregulated in the tumor tissues of patients with HCC compared with the corresponding adjacent normal tissues. However, the relationship between USP12 and the growth of HCCs is not fully understood. In the present study, USP12 was knocked down in HCC cell lines to investigate its effects on proliferation and apoptosis. The results showed that USP12-knockdown could inhibit the proliferation and promote apoptosis in HCC cell lines. Flow cytometry analysis also showed that USP12 could induce cell cycle arrest at the $\mathrm{G}_{2} / \mathrm{M}$ stage. In vivo experiments showed that USP12-knockdown could suppress tumor growth in mice, and immuno-blotting revealed that USP12 could induce $\mathrm{G}_{2} / \mathrm{M}$ arrest through the cyclin dependent kinase $1 /$ cyclinB1 axis, and trigger apoptosis via the p38/mitogen-activated protein kinase pathway. These data strongly indicate that USP12 is a potential target for the treatment of HCC.
\end{abstract}

\section{Introduction}

Hepatocellular carcinoma (HCC) is the most common type of primary liver cancer in adults (1). Worldwide, the number of new HCC diagnoses has increased annually, making HCC the second most common cause of cancer-related mortality (2). The survival rate of patients with HCC remains $<30 \%$ (3). Viral infection, alcoholism, obesity and diabetes are the major risk factors of HCC (4), which result in chronic inflammation,

Correspondence to: Professor Yong Lu, School of Laboratory Medicine, Wannan Medical College, 22 Wenchang West Street, Wuhu, Anhui 241002, P.R. China

E-mail: 1cs18755320789@163.com

${ }^{*}$ Contributed equally

Key words: ubiquitin specific protease 12, hepatocellular carcinoma, p38/mitogen-activated protein kinase pathway leading to the destruction and regeneration of hepatocytes, as well as genetic mutations and dysregulation of growth signals $(5,6)$. Since its etiology is so complex, monotherapy has been shown to be insufficient to cure all types of HCC (7).

The ubiquitin proteasome system (UPS) plays an important role in biological processes, especially in tumor cells (8), and $~ 95$ putative deubiquitinating enzymes have been identified in humans (9). As the reverse process of ubiquitination, deubiquitination also serves an important role $(10,11)$.

Ubiquitin-specific protease 12 (USP12) belongs to the UPS family, and promotes tumor pathogenesis through deubiquitination $(12,13)$. The WD repeat-containing protein, WDR48, in combination with USP12, suppresses AKT signaling by deubiquitinating $\mathrm{PH}$ domain leucine-rich repeat-containing protein phosphatase (PHLPP) in HCT116 cells. Overexpression of USP12 induces apoptosis and suppresses the proliferation of HCT116 cells (14). Also, USP12 regulates prostate cancer (PC) cells via the AKT/PHLPP pathway, and USP12-knockdown reduces cell proliferation, increases apoptosis and causes $\mathrm{G}_{1}$ arrest in PC cells $(15,16)$.

The cell cycle consists of a series of events, where cellular components are doubled and then accurately segregated into daughter cells. In eukaryotes, DNA replication is confined to discrete synthesis in the S-phase, and chromosomal segregation takes place at mitosis (the M-phase). The S- and M-phases are separated by two 'gap' phases, called $G_{1}$ and $G_{2}$. During these periods, cells acquire mass, integrate growth signals, arrange a replicated genome, and prepare for chromosomal segregation (17). Such ordered progression is tightly regulated by cell cycle checkpoints, at which the cell actively pauses proliferation until earlier processes, such as DNA replication or mitosis, are completed (18). These mechanisms play an indispensable role in maintaining genomic integrity in response to endogenous and exogenous DNA damage (19).

\section{Materials and methods}

Tissue array and immunohistochemistry. HCC tissue arrays were obtained from the National Engineering Center for Biochips. Immunohistochemical staining was conducted to examine USP12 expression in the tissues using a specific antibody against USP12. Briefly, the tissues were fixed with $4 \%$ 
paraformaldehyde overnight at room temperature, embedded with paraffin and serially cut at $4 \mu \mathrm{m}$. The sections were deparaffinized as follows: Xylene $10 \mathrm{~min}$, xylene $10 \mathrm{~min}$, absolute ethyl alcohol $5 \mathrm{~min}, 95 \%$ ethanol $5 \mathrm{~min}, 90 \%$ ethanol $5 \mathrm{~min}, 80 \%$ ethanol $5 \mathrm{~min}, 70 \%$ ethanol $5 \mathrm{~min}$, water $3 \mathrm{~min}$ and treated by sodium citrate, and then blocked with $3 \% \mathrm{H}_{2} \mathrm{O}_{2}$. Sections were incubated with the rabbit anti-USP12 primary antibody (1:150, cat. no. ab89870, Abcam) at $4^{\circ} \mathrm{C}$ overnight. Sections were then treated with peroxidase-conjugated secondary antibody (1:2,000, cat. no. ab205718, Abcam) and DAB chromogen. Then the samples were counterstained with hematoxylin, and observed under light microscope. The expression level was reflected by the staining intensity and the proportion of positively-stained cells, Positive reactions were defined as the presence of brown staining in the cell cytoplasm, nucleus and membrane. For USP12, a staining index (values 0-12) was determined by multiplying the score for staining intensity ( 0 , no staining; 1 , weak staining; 2 , moderate staining; and 3 , strong staining) by the score for the positive stained area (1, positive staining in $0-25 \%$ of tumor cells; 2 , positive staining in $>25-50 \%$ of tumor cells; 3 , positive staining in $51-75 \%$ of tumor cells; 4 , positive staining in $>75-100 \%$ of tumor cells). Finally, scores of 0-4 were considered low expression (-), and scores of 5-12 were considered high expression $(+)(20,21)$.

Cell culture. HCC cell lines (Huh7 and Hep3b) and 293T cells were purchased from the Shanghai Institutes of the Chinese Academy of Sciences. All cells were maintained in DMEM high glucose (Gibco; Thermo Fisher Scientific, Inc.) containing $10 \%$ fetal bovine serum (Gibco; Thermo Fisher Scientific, Inc.), $100 \mathrm{U} / \mathrm{ml}$ penicillin and $100 \mu \mathrm{g} / \mathrm{ml}$ streptomycin (complete medium) at $37^{\circ} \mathrm{C}$ in a humidified atmosphere containing $5 \%$ $\mathrm{CO}_{2}$. SB202190 (MedChemExpress), an inhibitor of the p38 pathway, was added into the cells at $20 \mu \mathrm{M}$ concentration and incubated for $24 \mathrm{~h}$ to determine the effect of USP12 on the p38 pathway.

Cell proliferation analysis. Cell proliferation was determined by multiparametric high content screening. HCC cells were infected with either negative control (NC) or USP12 short hairpin RNA (shRNA, 5'-CCGGCCAGATGTCTTACTTGT GAAACTATTCTCGAGAATAGTTTCACAAGTAAGACA TCTGGTTTTTG-3') lentiviruses (Shanghai GeneChem Co., Ltd.). Transfected cells were seeded into 96-well plates at a density of $2 \times 10^{3}$ cells/well, followed by incubation in complete medium at $37^{\circ} \mathrm{C}$ for 5 days. The Cellomics assay was carried out on an ArrayScan High Content Platform (cat. no. ASN00004F; Thermo Fisher Scientific, Inc.). The infected cells were identified, and the intensity and distribution of fluorescence in each cell were reported (>800 cells were examined). The acquired images and obtained data were stored in a Microsoft SQL database (https://www.compuwork. ca/canada/bc/vancouver/database/expert/microsoft/SQL_Server.aspx).

USP12-knockdown in HCC cell lines. The lentiviral vector pGCSIL-GFP (Shanghai GeneChem Co., Ltd.) was digested using the restriction enzymes $A g e I$ and EcoRI. Subsequently, small interfering RNA (siRNA) targeting the USP12 sequence (knockdown, 5'-CCAGAUGUCUUACUUGUG AAACUAU-3') and a non-silencing sequence (5'-TTCTCC
GAACGTGTCACGT-3') were transformed into shRNA (stem-loop-stem structure), followed by cloning into the predigested lentiviral vector. The recombinant plasmid and two viral plasmids (Shanghai GeneChem Co., Ltd.) were transfected into the human 293 T cells using Lipofectamine ${ }^{\circledR} 3000$ (Invitrogen; Thermo Fisher Scientific, Inc.) according to the manufacturer's protocols. Transfected cells were incubated for 3 days at $37^{\circ} \mathrm{C}$, and lentiviral particles was collected from the culture medium. For stable infection, 10,000 HCC cells were seeded into 6-well plates, followed by infection with the USP12 shRNA-expressing (USP12-shRNA) or non-silencing shRNA-expressing lentivirus (control) at a multiplicity of infection of 10 .

Tumor xenografts. A total of 10 female BALB/c nude mice (4-6 weeks old, 18-20 g weight) were purchased from the Model Animal Research Centre of Nanjing University. The animals were housed in a purpose-built designated pathogen-free facility under standard conditions maintained in standard conditions (temperature, $18-22^{\circ} \mathrm{C}$; humidity, 50-60\%; 12-h light/dark cycle). The mice were provided with ad libitum access to food and water. The animal-related protocols were approved by the Institutional Animal Care and Use Committee of Wannan Medical College. Briefly, cell suspensions containing $2 \times 10^{6}$ tumor cells in $0.2 \mathrm{ml}$ serum-free DMEM (Gibco; Thermo Fisher Scientific, Inc.) were subcutaneously injected into the flank of each mouse. Tumor cells containing USP12-shRNA or control tumor cells were injected into the right flank (five mice in each group). Tumor growth was recorded by determining the length (L) and width (W) of each tumor with a caliper, and the formula $\mathrm{Lx} \mathrm{W}^{2} \mathrm{x}(\pi / 6)$ was employed to calculate the tumor size. If the diameter of the largest tumor was $>15 \mathrm{~mm}$, the mice were deemed as ineffective, although this did not occur in the present study. The total duration of the xenograft experiment was 22 days, and the mice were anesthetized by the intraperitoneal injection of pentobarbital sodium $(60 \mathrm{mg} / \mathrm{kg})$ and then euthanized by carbon dioxide asphyxiation at the end of the experiment. The flow rate of carbon dioxide used for euthanasia did not displace $>30 \%$ of the chamber volume/minute.

Flow cytometry analysis. Cells $\left(1 \times 10^{6}\right)$ undergoing lentiviral transfection were harvested and washed twice with cold PBS, followed by fixation in cold $70 \%$ ethanol overnight. Fixed cells were resuspended in PBS, and the cell suspension was filtered through a 400-mesh membrane. The cells were stained with propidium iodide (PI) (eBioscience; Thermo Fisher Scientific, Inc.) for $1 \mathrm{~h}$ at room temperature for cell cycle analysis, and then analyzed using a BD FACSCalibur flow cytometer (BD Biosciences). Each experiment was carried out at least in triplicate.

Western blotting. Tumor cells were lysed in RIPA buffer (Nanjing KeyGen Biotech Co., Ltd., cat. no. KGP702) containing fresh $1 \mathrm{mM}$ protease inhibitor (Nanjing KeyGen Biotech Co., Ltd., cat. no. KGP603) and $1 \mathrm{mM}$ phosphatase inhibitor (Nanjing KeyGen Biotech Co., Ltd., cat. no. KGP602). The protein concentration was measured using a bicinchoninic acid assay kit (Pierce; Thermo Fisher Scientific, Inc.). Equal amounts of protein $(20 \mu \mathrm{g})$ were subjected to $10 \%$ SDS-PAGE 
Table I. Expression of USP12 in human liver cancer tissue array.

USP12 expression

\begin{tabular}{|c|c|c|c|c|}
\hline & & & & \\
\hline Characteristic & Patients $(n=90)$ & High & Low & P-value \\
\hline Gender & & & & 0.890 \\
\hline Male & 53 & 28 & 25 & \\
\hline Female & 37 & 19 & 18 & \\
\hline Age & & & & 0.203 \\
\hline$\leq 65$ & 40 & 23 & 17 & \\
\hline$>65$ & 50 & 22 & 28 & \\
\hline Tumor Size & & & & 0.209 \\
\hline$\leq 5 \mathrm{~cm}$ & 44 & 20 & 24 & \\
\hline$>5 \mathrm{~cm}$ & 46 & 27 & 19 & \\
\hline Differentiation & & & & $0.034^{\mathrm{a}}$ \\
\hline $\mathrm{I} / \mathrm{II}$ & 32 & 13 & 19 & \\
\hline III & 58 & 39 & 19 & \\
\hline TNM Stage & & & & 0.249 \\
\hline $\mathrm{I} / \mathrm{II}$ & 38 & 18 & 20 & \\
\hline III/IV & 52 & 31 & 21 & \\
\hline Location & & & & $<0.0001^{\mathrm{b}}$ \\
\hline Tumor tissue & 90 & 67 & 23 & \\
\hline Adjacent tissue & 90 & 31 & 59 & \\
\hline
\end{tabular}

A tissue microarray was stained with anti-human USP12 antibody. ${ }^{\mathrm{a}} \mathrm{P}<0.05$; ${ }^{\mathrm{b}} \mathrm{P}<0.001$. USP12, ubiquitin-specific protease 12 .

and then electro-transferred onto a PVDF membrane. The blot was incubated in $10 \mathrm{mM}$ Tris- $\mathrm{HCl}$ ( $\mathrm{pH} 7.4)$, consisting of $3 \%$ BSA (Nanjing KeyGen Biotech Co., Ltd., cat. no. KGY00810) and $0.05 \%$ Tween-20 at room temperature for $2 \mathrm{~h}$, to block non-specific bindings. Subsequently, the membrane was incubated with primary antibody at $4^{\circ} \mathrm{C}$ for $12 \mathrm{~h}$, followed by incubation with a corresponding peroxidase-conjugated secondary antibody (Santa Cruz Biotechnology) at room temperature for $2 \mathrm{~h}$. The immunoreactive bands were visualized using the SuperSignal West Pico Chemiluminescent Substrate (Pierce; Thermo Fisher Scientific, Inc.), and the band density was quantified by densitometric analysis and using GAPDH as normalization control by a Versadoc Imaging System Model 3000 (Bio-Rad Laboratories, Inc.) at room temperature for $1 \mathrm{~min}$. The details of primary and secondary antibodies used in the experiment are shown in Table SI. The experiment was conducted $\geq 3$ times.

Reverse transcription-quantitative PCR (RT-qPCR). Total RNA was extracted from the HCC cell lines using TRIzol ${ }^{\circledR}$ (Invitrogen; Thermo Fisher Scientific, Inc.) following the manufacturer's instructions. RT-qPCR was performed as reported previously (7). Briefly, $1 \mu \mathrm{g}$ of total RNA was reverse transcribed using random primers and Primescript reverse transcriptase (Vazyme). qPCR reactions for the indicated genes were carried out using the SYBR-Green qPCR kit (Vazyme) on a fluorescent temperature cycler (ABI 7500 Real-Time PCR system; Thermo Fisher Scientific, Inc.). The following primers were used to detect the expression of USP12

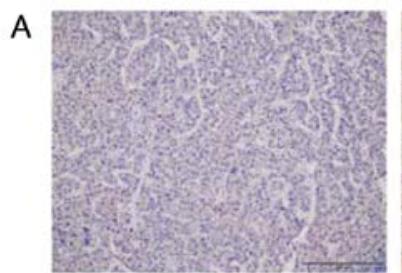

Normal tissues

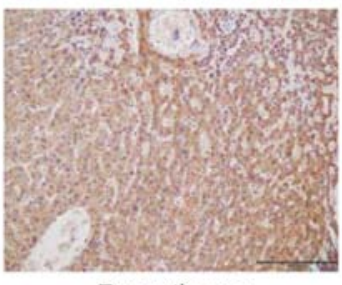

Tumor tissues

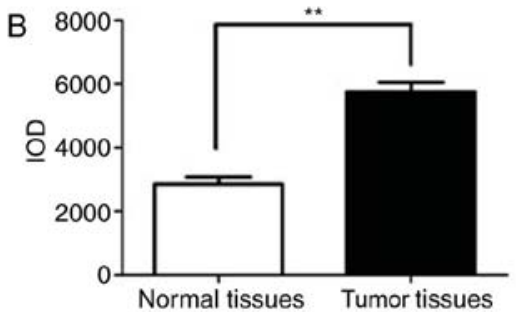

Figure 1. Immunohistochemical staining of USP12 expression in human tissue microarrays. (A) Representative staining of USP12 expression in tumor tissues and corresponding adjacent normal tissues. Normal liver tissues showed weak/undetectable USP12 staining, while hepatocellular carcinoma tissues showed high USP12 staining. Scale bar $=200 \mu \mathrm{m}$. (B) IOD analysis of USP12 staining in cancer tissues and normal tissues. ${ }^{* *} \mathrm{P}<0.01$, as indicated. USP12, ubiquitin-specific protease 12 , IOD, integrated optical density.

forward, 5'-AGACCTTTCTGTTGACGTGGA-3' and reverse, 5'-TGTTTGCTGCGACACTCTTC-3'; and GAPDH forward, 5'-TGACTTCAACAGCGACACCCA-3' and reverse, 5'-CAC CCTGTTGCTGTAGCCAAA-3'. Briefly, after an initial 
A

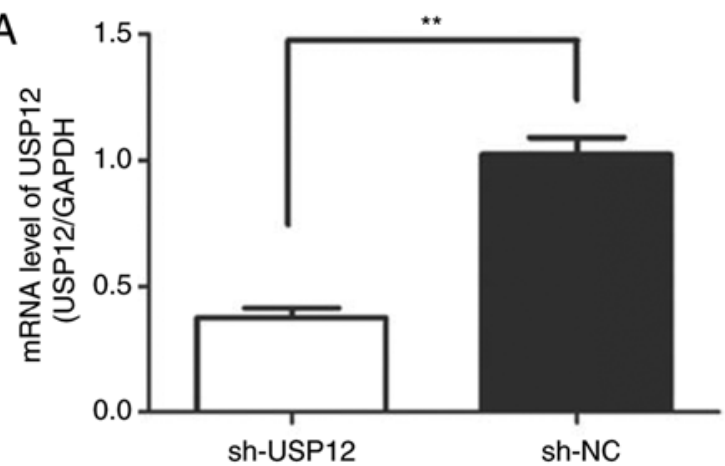

Huh7 cells

C
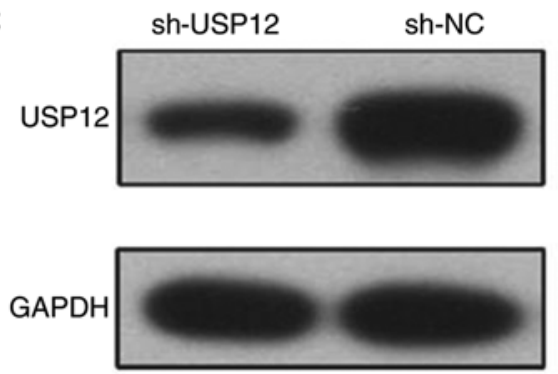

Huh7 cells

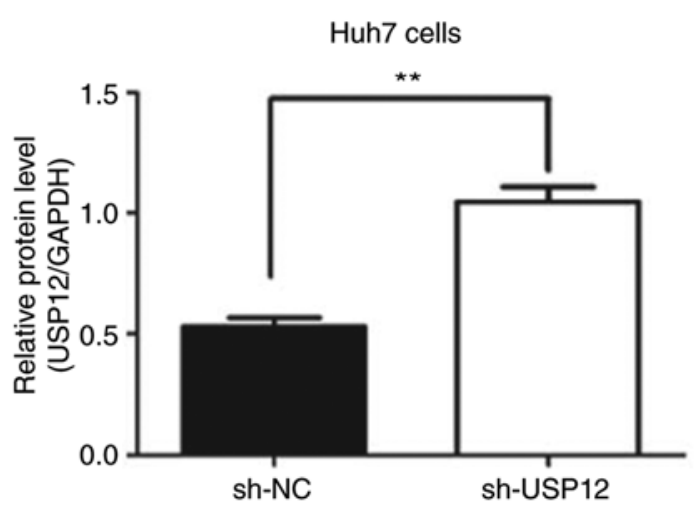

B

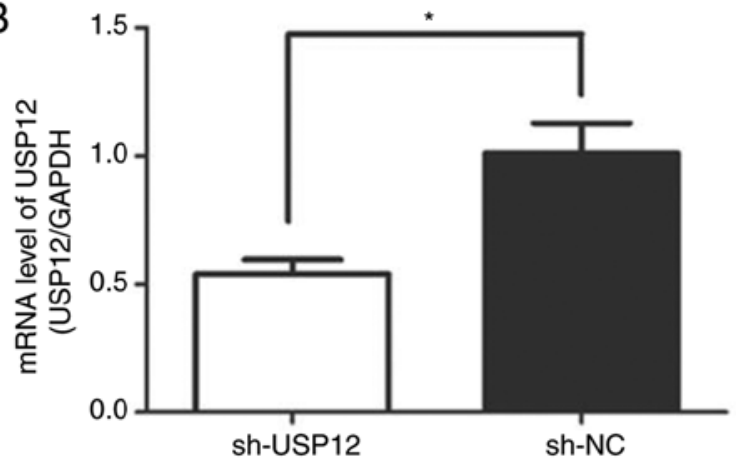

Hep3b cells
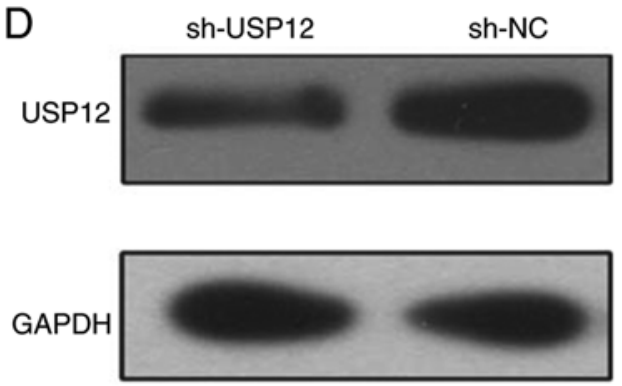

Hep3b cells

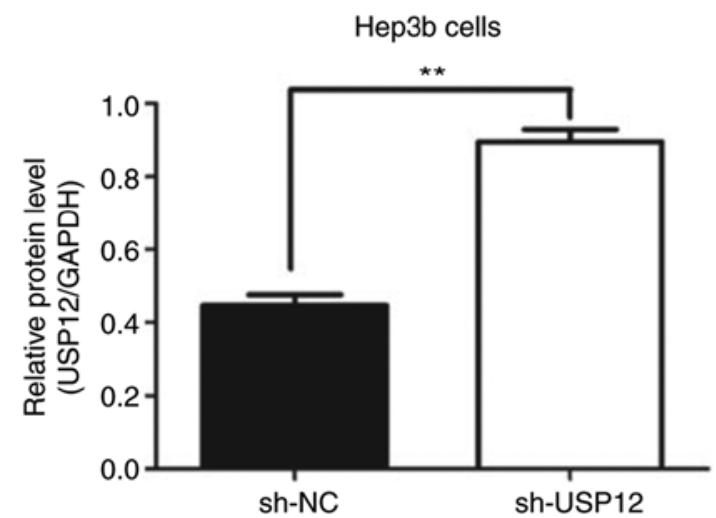

Figure 2. mRNA and protein level determination of USP12 in hepatocellular carcinoma cell lines after USP12 knockdown. (A and B) The mRNA level of USP12 was examined by reverse transcription-quantitative PCR in Huh7 and Hep3b cells. (C and D) The protein level of USP12 was examined by western blot analysis, and GAPDH was used as an internal control. ${ }^{~} \mathrm{P}<0.05$ and ${ }^{* *} \mathrm{P}<0.01$, as indicated. USP12, ubiquitin-specific protease 12 ; sh, short hairpin; NC, negative control.

denaturation step at $95^{\circ} \mathrm{C}$ for $5 \mathrm{~min}, 45$ cycles of amplification were carried out with 45 cycles at $95^{\circ} \mathrm{C}$ for $15 \mathrm{sec}$, and an annealing temperature of $60^{\circ} \mathrm{C}$ for $1 \mathrm{~min}$. GAPDH was selected as the housekeeping gene, and the relative gene expression of the target gene was determined by the $2^{-\Delta \Delta C q}$ method (13). The experiment was repeated $\geq 3$ times.

Statistical analyses. All statistical analyses were performed using SPSS 18.0 software (SPSS, Inc.) and the data were expressed as mean \pm standard deviation. Differences among categorical variables were analyzed using the $\chi^{2}$ test. The expression levels of USP12 in xenografted tumors were analyzed by the independent sample Student's t-test. The immunoreactive scores for USP12 for tissue arrays were analyzed using the non-parametric Mann-Whitney U, Kruskal-Wallis $\mathrm{H}$ and Wilcoxon tests followed by the Dunn's test post hoc test. Multiple groups were analyzed with one-way ANOVA followed by the Tukey post hoc test. $\mathrm{P}<0.05$ was considered to indicate a statistically significant difference.

\section{Results}

USP12 expression in human HCC tissues. The array consisted of $90 \mathrm{HCC}$ patient tissue samples, including tumor tissues and paired-adjacent normal tissues. Of these 90 patients, 53 were male and 37 female, with a median age of 68 years (range, 25-87 years). Table I and Fig. 1A show that USP12 was highly expressed in HCC tumor tissue samples By contrast, the USP12 expression in adjacent normal tissue samples was significantly lower than that in the tumor tissues (Table I; $\mathrm{P}<0.001)$. Moreover, USP12 high expression may indicated poor differential HCC, and the expression level of USP12 was not associated with other factors. However, integrated optical density analysis revealed that the USP12 expression was lower 
A
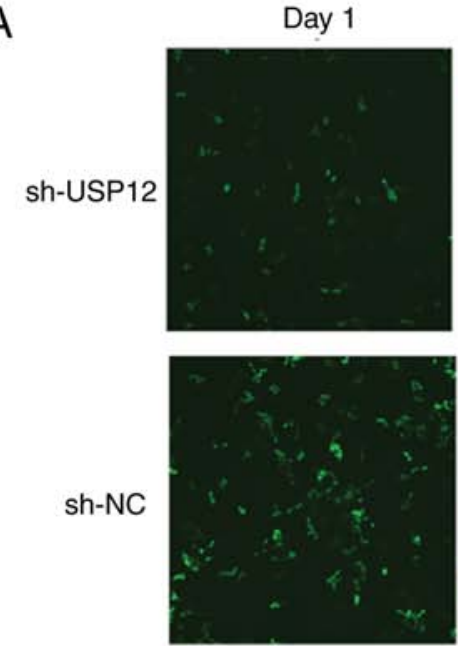

B

sh-USP12
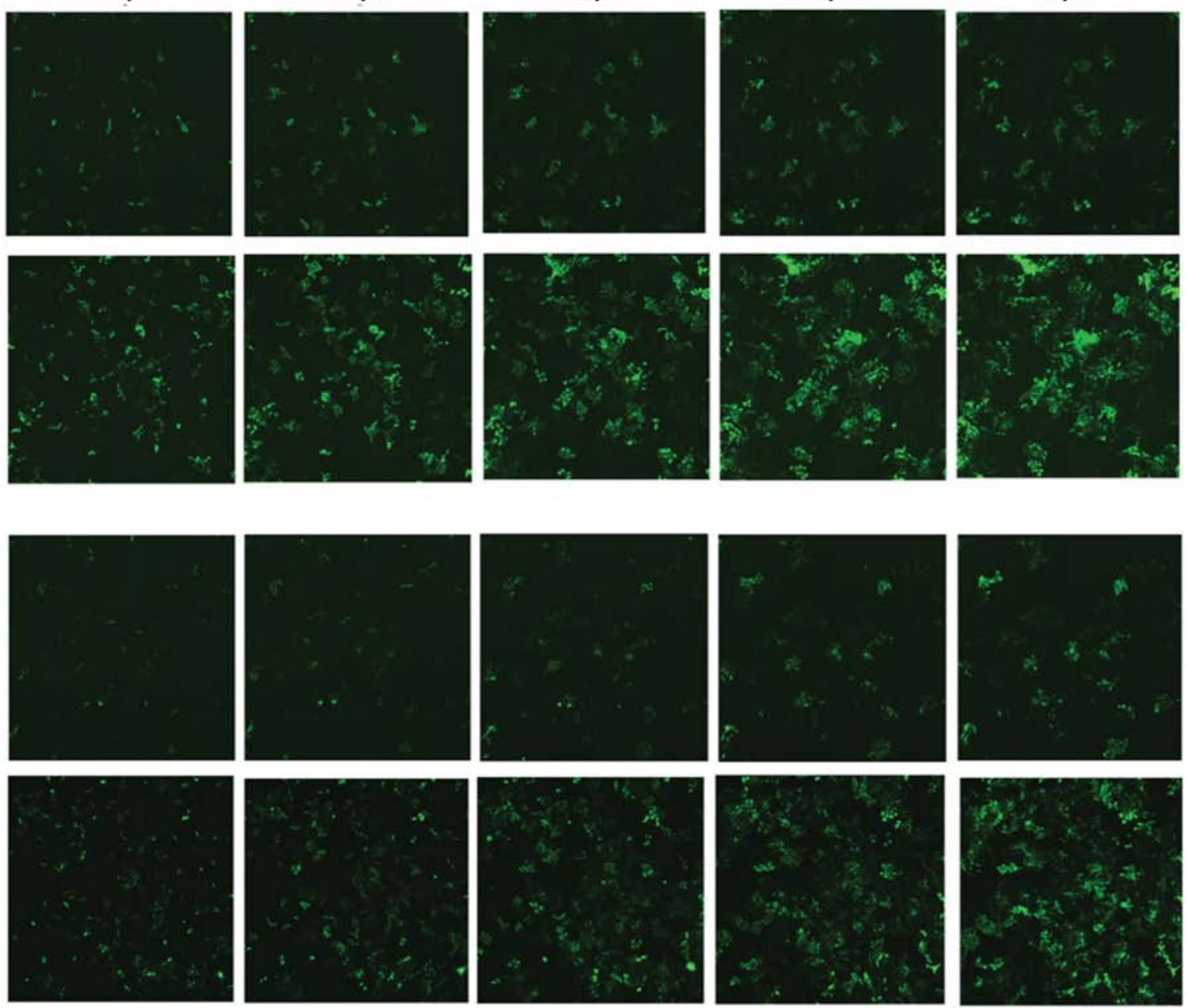

sh-NC
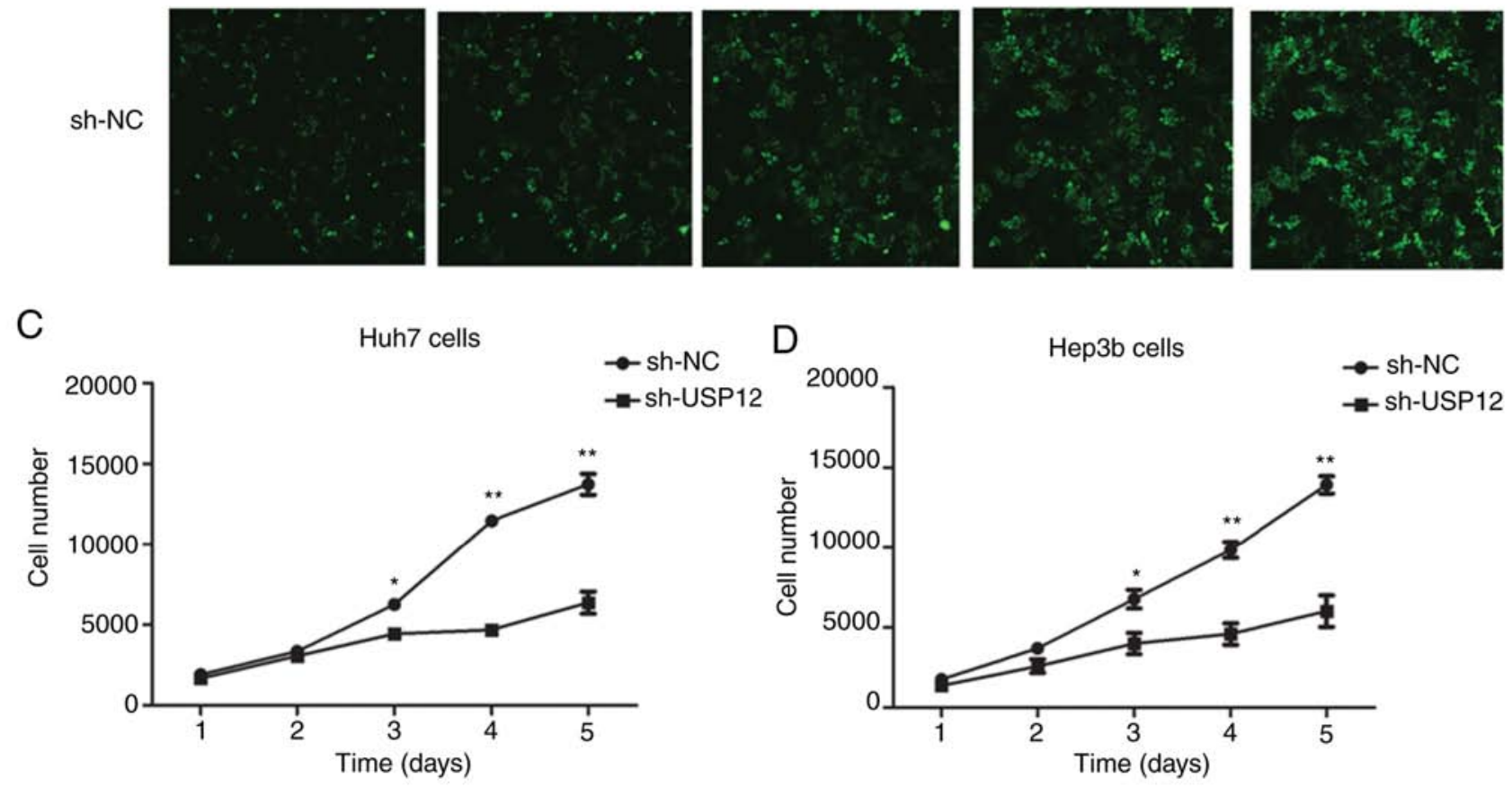

Figure 3. Effect of downregulation of USP12 on hepatocellular carcinoma cell growth. USP12-shRNA-targeted and negative control (A) Huh7 and (B) Hep3b cells were observed by Cellomics for 5 days. Cell count curve of USP12-shRNA targeted cells and negative control cells in (C) Huh7 and (D) Hep3b cells. ${ }^{*} \mathrm{P}<0.05$ sh-NC vs. sh-USP12 in Huh7 cells and Hep3b cells at day 3 and ${ }^{* *} \mathrm{P}<0.01$ sh-NC vs. sh-USP12 at days 4 and 5. USP12, ubiquitin-specific protease 12; shRNA, short hairpin RNA; sh, short hairpin; NC, negative control.

in tumor tissues compared with that in corresponding normal tissues (Fig. 1B).

Efficiency of USP12-knockdownin HCC cell lines in vitro. In the present study, USP12 expression in HCC cell lines was knocked down by shRNA in order to assess its role in the tumorigenesis of HCC. Fig. 2A and C show that the USP12 expression at both the mRNA and protein levels were significantly decreased in the target cells compared with those in the control cells after transfection in Huh7 cells; a similar effect was observed in Hep3b cells (Fig. 2B and D).

USP12-knockdown inhibits the proliferation of HCC cell lines in vitro. To investigate the effect of USP12 on cell proliferation, HCC cells transfected with either the USP12-shRNA or the $\mathrm{NC}$ lentiviral vector were examined by Cellomics assay. The 

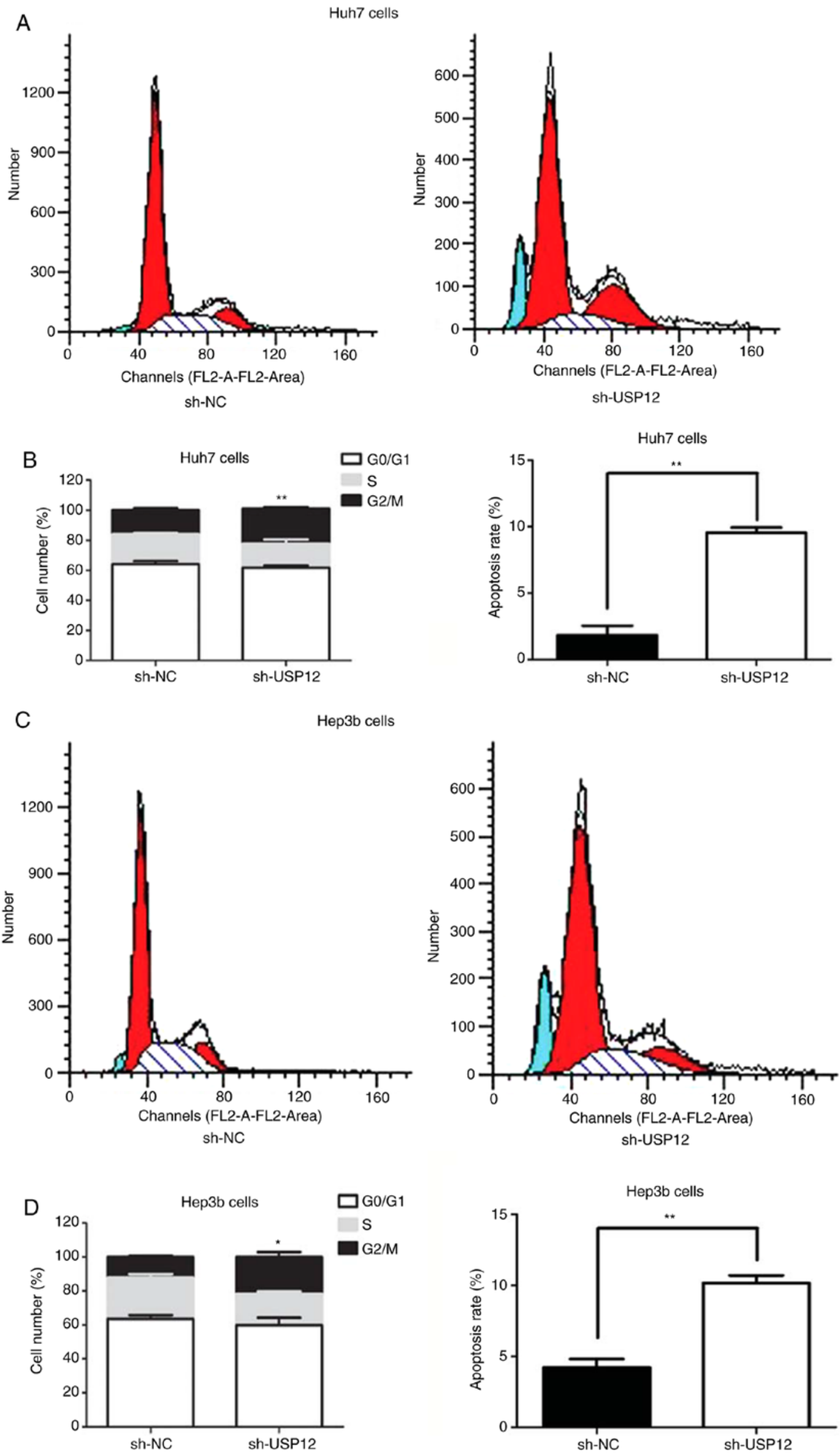

Figure 4. Influence of USP12-knockdown on the hepatocellular carcinoma cell cycle. The percentage of cells in the $\mathrm{G}_{2} / \mathrm{M}$ phase was remarkably increased after USP12-knockdown in (A and B) Huh7 and (C and D) Hep3b cells analyzed by flow cytometry. ${ }^{*} \mathrm{P}<0.05$ sh-NC vs. sh-USP12, ${ }^{* *} \mathrm{P}<0.01$ sh-NC vs. sh-USP12. USP12, ubiquitin specific protease 12; sh, short hairpin; NC, negative control. 
A
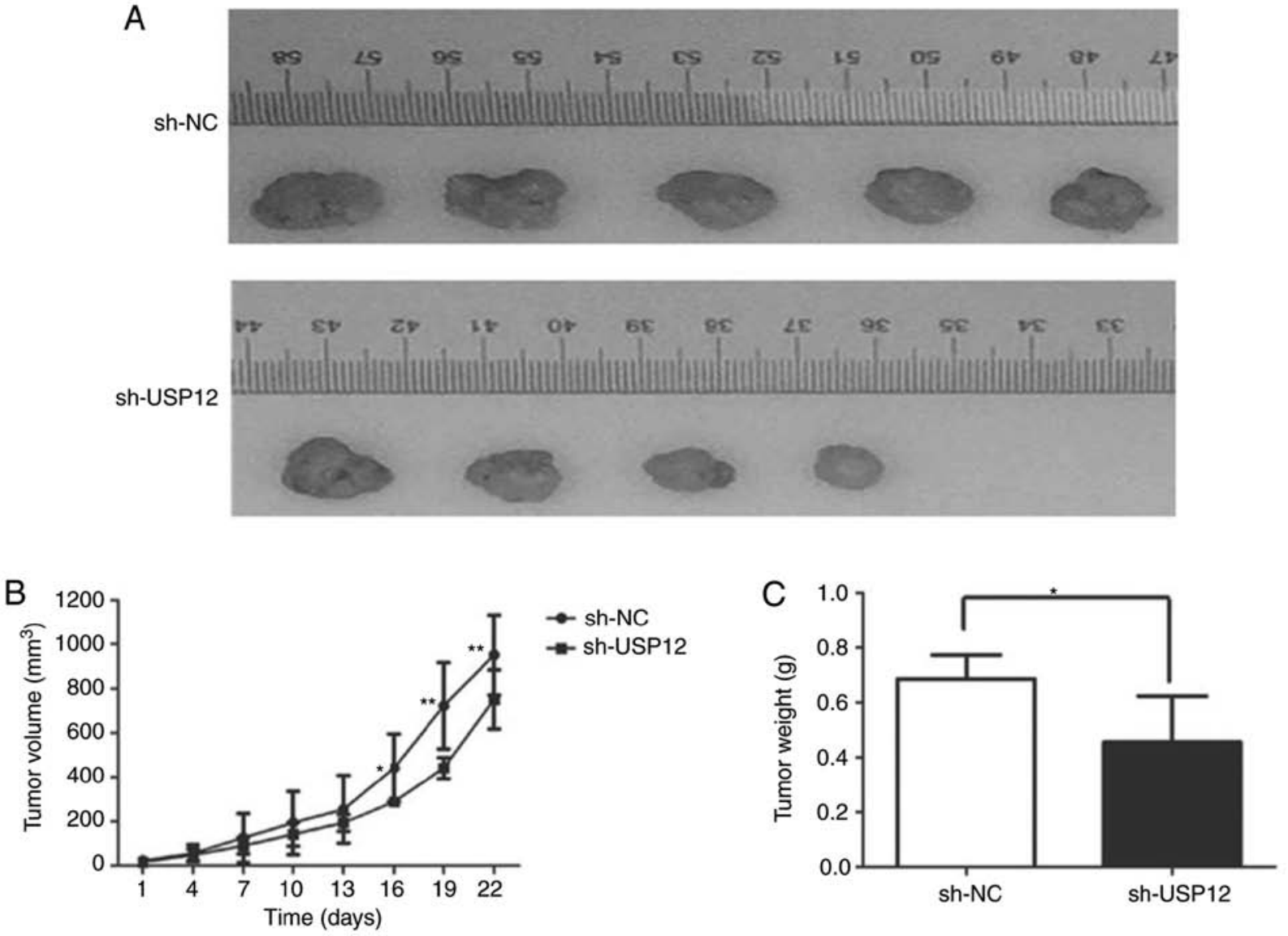

Figure 5. USP12-knockdown inhibits the growth of Huh7 cells in vivo. (A) USP12-knockdown in Huh7 cells decreased xenografted tumor growth in nude mice. Each group contained five mice, but one mouse in the USP12-knockdown group died seven days after injection. The data were collected 22 days after the tumor was first observed. (B) Tumor growth was significantly inhibited after USP12-knockdown. ${ }^{*} \mathrm{P}<0.05$ sh-NC vs. sh-USP12 at day $16,{ }^{* *} \mathrm{P}<0.01$ sh-NC vs sh-USP12 at day 19 and 22. (C) The weight of the tumor was significantly decreased after USP12-knockdown. ${ }^{*}<0.05$ sh-NC vs. sh-USP12. USP12, ubiquitin specific protease 12; sh, short hairpin; NC, negative control.

proliferation of HCC cells containing NC or USP12-shRNA was determined each day for 5 days (Fig. 3A and B). The results revealed that proliferation was inhibited following USP12-knockdown (Fig. 3C and D). Therefore, these findings suggest that USP12-knockdown suppressed the proliferation of HCC cells.

The regulatory role of USP12 in the proliferation of HCC cells was also assessed by flow cytometry. The results indicated that USP12-knockdown resulted in a reduced proportion of cells in the $\mathrm{G}_{0} / \mathrm{G}_{1}$ phase and an increased proportion in the $\mathrm{G}_{2} / \mathrm{M}$ phase, and that apoptosis was increased after USP12-knockdown (Fig. 4A and D). These results suggest that USP12 induces apoptosis and $\mathrm{G}_{2} / \mathrm{M}$ arrest in $\mathrm{HCC}$ cells.

USP12-knockdown inhibits the growth of HCC cells in vivo. To explore the mechanisms underlying the regulatory effect of USP12 on cell proliferation in vivo, Huh7 cells containing USP12 shRNA or non-silencing RNA were injected into nude mice. The weight and volume of tumors were significantly decreased after USP12-knockdown, which suggests that USP12-knockdown is associated with decreased tumor growth (Fig. 5).

USP12 induces apoptosis via the mitogen-activated protein kinase (MAPK) pathway, and inhibits the proliferation of $\mathrm{HCC}$ cells, via $G_{2} / M$ arrest. Since the apoptotic rate of
HCC cells was significantly increased after USP12-knockdown, it was hypothesized that USP12 regulated cell growth via the p38/MAPK pathway. Phosphorylated p38 (p-p38) and phosphorylated JNK (p-JNK) were activated after USP12-knockdown, and inhibition of the MAPK pathway could eliminate such activation. Furthermore, the apoptosis markers, cleaved-caspase 9 and cleaved-caspase 3, were upregulated following USP12-knockdown (Fig. 6A). Therefore, it was concluded that USP12 regulated the proliferation of HCC cells, at least partly, through the p38/MAPK pathway. The MAPK pathway is an important pathway in the cell cycle, and it can induce a wide variety of downstream events, including apoptosis and $\mathrm{G}_{2} / \mathrm{M}$ arrest (22). The molecular mechanisms underlying the regulatory effect of USP12 on cell proliferation were further investigated and the expression of cell cycle proteins was determined. Fig. 6B displays that the expression of M-phase inducer phosphatase 3 (Cdc25c), Cdk1 and cyclin B1 were all decreased after USP12-knockdown. Fig. 6B shows that inhibition of the p38/MAPK pathway rescued the activation of the p38/MAPK pathway induced by the downregulation of USP12.

\section{Discussion}

As one of the most commonly detected tumors worldwide, HCC has a high mortality rate, particularly in China (23). Identification 
A
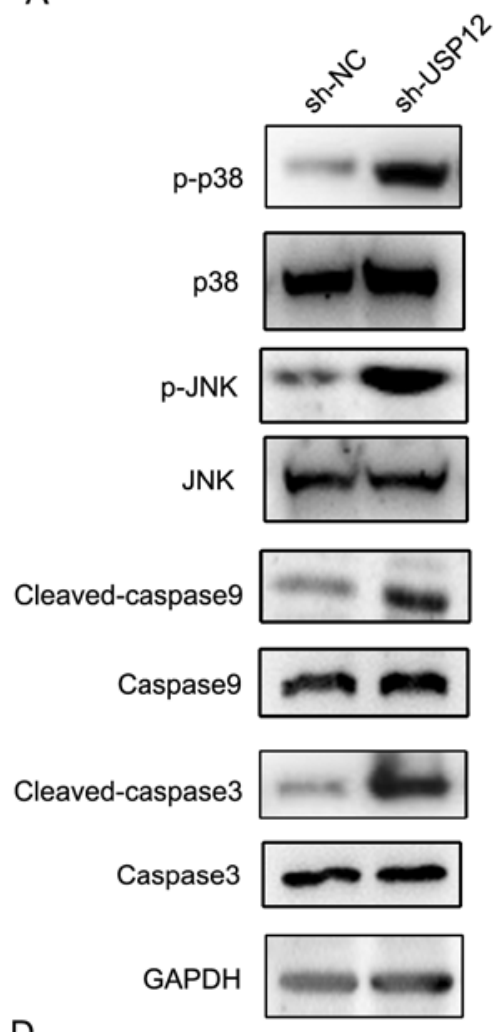

D

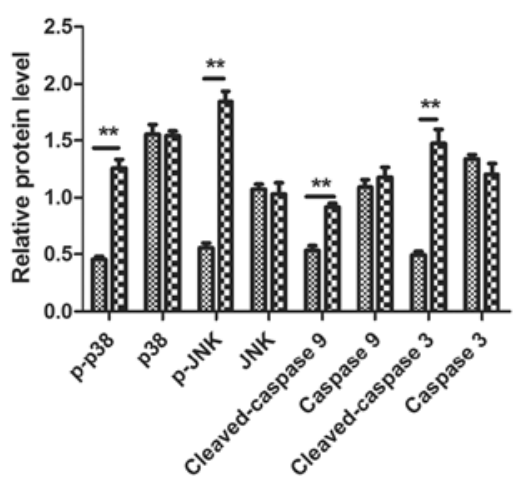

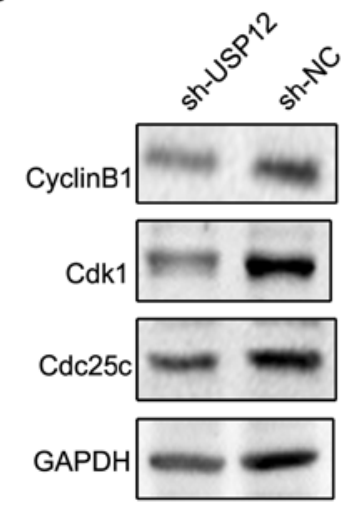

E
C

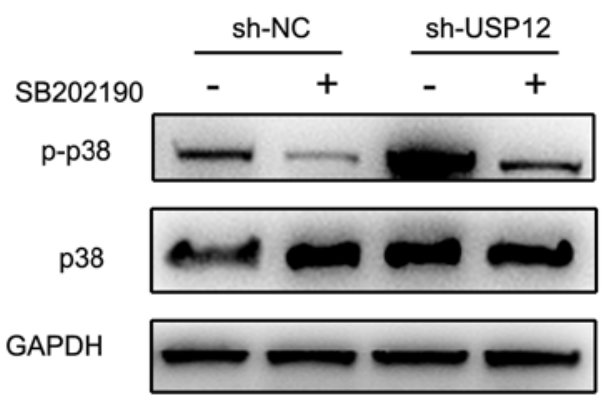

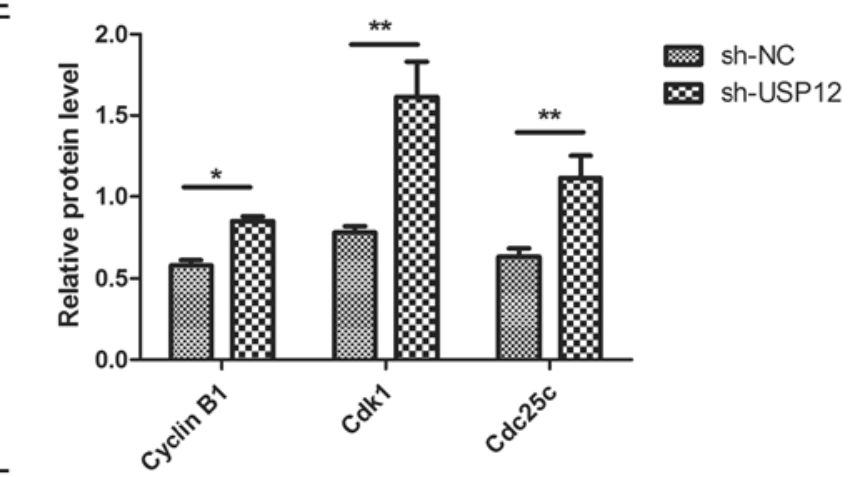

$\mathrm{F}$

sh-NC

sh-USP12

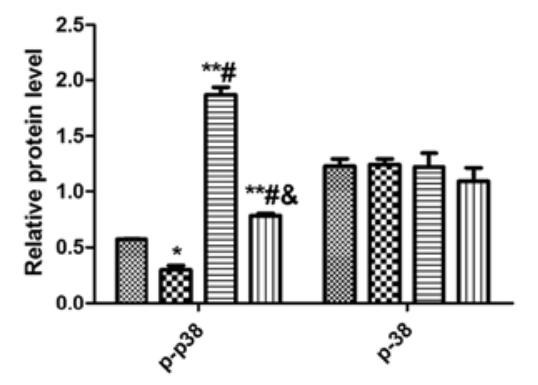

sh-NC/SB202190-

$\infty$ sh-NC/SB202190+

曰 sh-USP12/SB202190-

m sh-USP12/SB202190+

Figure 6. Mechanisms of USP12 regulation of tumor growth and apoptosis. Western blot analysis was carried out to detect the expression of cell cycle checkpoint proteins and apoptosis markers in Huh7 cells. (A) The level of p-p38 and p-JNK were upregulated while the expression levels of p38 and JNK were not changed after USP12-knockdown. The levels of cleaved-caspase 9 and cleaved-caspase 3 were upregulated after USP12-knockdown, while caspase 9 and caspase 3 were not changed after USP12-knockdown. (B) The level of cyclin B1, Cdk1 and Cdc25c was decreased after USP12-knockdown. GAPDH was used as an internal control. (C) The level of p-p38 was downregulated after addition of SB202190 (20 $\mu$ M), an inhibitor of the p38/MAPK pathway. SB202190 decreased the upregulation of p-p38 after USP12-knockdown. (D) Statistical analysis of (A), ${ }^{* *} \mathrm{P}<0.01$ vs. the corresponding group. (E) The statistical analysis of (B), ${ }^{*} \mathrm{P}<0.05$ vs. the corresponding group, ${ }^{* *} \mathrm{P}<0.01$ vs. the corresponding group. $(\mathrm{F})$ The statistical analysis of $(\mathrm{C}),{ }^{*} \mathrm{P}<0.05$ vs. sh-NC/SB202190-, ${ }^{* *} \mathrm{P}<0.01 \mathrm{vs}$. sh-NC/SB202190-. " P<0.01 vs. sh-NC/SB202190+ and ${ }^{\circledR} \mathrm{P}<0.01$ vs. sh-USP12/SB202190-. USP12, ubiquitin-specific protease 12; p-, phosphorylated; Cdc25c, M-phase inducer phosphatase 3; MAPK, mitogen-activated protein kinase; sh, short hairpin; NC, negative control.

of novel target genes for further research is in progress. The present study found that USP12 expression was higher in human HCC tissues compared with the adjacent normal tissues. In vitro and in vivo experiments showed that USP12-knockdown inhibited the proliferation of HCC cells. Moreover, the molecular mechanisms underlying the regulatory role of USP12 were also investigated. Taken together, these findings provide evidence that USP12 may be used as a candidate for therapeutic intervention during HCC.

The correct timing of cell cycle events is regulated by cell-cycle checkpoints (24). Consequently, checkpoint blockade results in cell-cycle arrest and alters cellular proliferation. The results of the present study showed that USP12-knockdowninhibited cellular proliferation through cell cycle arrest. A previous study showed that USP12-knockdown may inactivate $\mathrm{G}_{0} / \mathrm{G}_{1}$ arrest in HeLa cells (25). A similar effect has also been observed in PC, and USP12 deficiency leads to decreased proliferative ability, as well as increased apoptosis and $\mathrm{G}_{1}$ arrest (15). The present study showed that USP12-knockdown induced $\mathrm{G}_{2} / \mathrm{M}$ arrest in $\mathrm{HCC}$ cell lines. In addition, USP12-knockdownincreased the levels of apoptosis in HCC cell lines. Furthermore, the effect of USP12 on the growth of HCC cells was also investigated in vivo. The results showed that tumor growth was significantly inhibited by USP12-knockdown.

Since the data from the present study showed that USP12-knockdown induced $\mathrm{G}_{2} / \mathrm{M}$ arrest and apoptosis, the 
expression of cell cycle checkpoint proteins was determined by western blotting. The results were consistent with cell cycle analysis; $\mathrm{Cdc} 25 \mathrm{c}, \mathrm{Cdk} 1$ and cyclin B1 were all significantly downregulated after USP12-knockdown. Previous studies have indicated that $\mathrm{Cdc} 25 \mathrm{c}$ can be inactivated by $\mathrm{p} 38$, leading to initiated $\mathrm{G}_{2} / \mathrm{M}$ cell cycle transition $(26,27)$, and that p38 activation can induce the apoptosis cascade (28-30). Furthermore, the present study also assessed the status of the p38/MAPK pathway, and found that it was activated by USP12-knockdown. It was also found that activation of the p38/MAPK pathway was reversed by SB202190. These results reveal that USP12 regulates the proliferation of HCC cells primarily via the p38/MAPK pathway.

Collectively, by using in vitro and in vivo approaches, the presentstudy provided evidence thatUSP12-knockdowninhibited tumor growth in human $\mathrm{HCC}$ by inducing $\mathrm{G}_{2} / \mathrm{M}$ arrest and apoptosis, at least partly, via the p38/MAPK pathway. In addition, USP12 expression was associated with the pathological tumor stage of HCC, suggesting that USP12 could be considered as a promising therapeutic target for $\mathrm{HCC}$.

\section{Acknowledgements}

We thank Dr Nanlin Jiao (Department of Pathology, Yijishan Hospital of Wannan Medical College) for the kind assistance in immunohistochemistry statistical analysis.

\section{Funding}

The present study was supported by the Key Project of Anhui University Natural Science Research (grant no. KJ2017A260).

\section{Availability of data and materials}

The datasets used and/or analyzed during the current study are available from the corresponding author on reasonable request.

\section{Authors' contributions}

CL, XL and MC performed the experiments. GF, FL and GZ analyzed the data. YL and CL designed the experiments and wrote the manuscript. All authors read and approved the final manuscript.

\section{Ethics approval and consent to participate}

The human samples used in the present study were purchased from Shanghai Outdo Biotech (Shanghai, China). The samples of this array were obtained from Taizhou Hospital of Zhejiang Province, and the use of this array was approved by the ethics committee of Taizhou Hospital of Zhejiang Province.

\section{Patient consent for publication}

Not applicable.

\section{Competing interests}

The authors declare that they have no competing interests.

\section{References}

1. Siegel RL, Miller KD and Jemal A: Cancer statistics, 2015. CA Cancer J Clin 65: 5-29, 2015.

2. Bellissimo F, Pinzone MR, Cacopardo B and Nunnari G: Diagnostic and therapeutic management of hepatocellular carcinoma. World J Gastroenterol 21: 12003-12021, 2015.

3. Goh GB, Chang PE and Tan CK: Changing epidemiology of hepatocellular carcinoma in Asia. Best Pract Res Clin Gastroenterol 29: 919-928, 2015.

4. Dutta R and Mahato RI: Recent advances in hepatocellular carcinoma therapy. Pharmacol Ther 173: 106-117, 2017.

5. Liu Z, Tu K and Liu Q: Effects of microRNA-30a on migration, invasion and prognosis of hepatocellular carcinoma. FEBS Lett 588: 3089-3097, 2014.

6. Li C, Chen J, Zhang K, Feng B, Wang R and Chen L: Progress and prospects of long noncoding RNAs (lncRNAs) in hepatocellular carcinoma. Cell Physiol Biochem 36: 423-434, 2015.

7. Makita Y, Murata S, Katou Y, Kikuchi K, Uejima H, Teratani M, Hoashi Y, Kenjo E, Matsumoto S, Nogami M, et al: Anti-tumor activity of KNTC2 siRNA in orthotopic tumor model mice of hepatocellular carcinoma. Biochem Biophys Res Commun 493: 800-806, 2017.

8. Ciechanover A: The ubiquitin-proteasome proteolytic pathway. Cell 79: 13-21, 1994

9. Eletr ZM and Wilkinson KD: Regulation of proteolysis by human deubiquitinating enzymes. Biochim Biophys Acta 1843: 114-128, 2014.

10. Sato Y, Yamagata A, Goto-Ito S, Kubota K, Miyamoto R, Nakada S and Fukai S: Molecular basis of Lys-63-linked polyubiquitination inhibition by the interaction between human deubiquitinating enzyme OTUB1 and ubiquitin-conjugating enzyme UBC13. J Biol Chem 287: 25860-25868, 2012.

11. Reyes-Turcu FE and Wilkinson KD: Polyubiquitin binding and disassembly by deubiquitinating enzymes. Chem Rev 109: 1495-1508, 2009.

12. Youle RJ and van der Bliek AM: Mitochondrial fission, fusion, and stress. Science 337: 1062-1065, 2012.

13. Komander D, Clague MJ and Urbé S: Breaking the chains: Structure and function of the deubiquitinases. Nat Rev Mol Cell Biol 10: 550-563, 2009.

14. McClurg UL, Summerscales EE, Harle VJ, Gaughan L and Robson CN: Deubiquitinating enzyme Usp12 regulates the interaction between the androgen receptor and the Akt pathway. Oncotarget 5: 7081-7092, 2014.

15. Burska UL, Harle VJ, Coffey K, Darby S, Ramsey H, O'Neill D, Logan IR, Gaughan L and Robson CN: Deubiquitinating enzyme Usp12 is a novel co-activator of the androgen receptor. J Biol Chem 288: 32641-32650, 2013.

16. Wei R, Liu X, Yu W, Yang T, Cai W, Liu J, Huang X, Xu GT, Zhao S, Yang J and Liu S: Deubiquitinases in cancer. Oncotarget 6: 12872-12889, 2015.

17. Stewart ZA and Pietenpol JA: Cell cycle checkpoints as therapeutic targets. J Mammary Gland Biol Neoplasia 4: 389-400, 1999.

18. Medema RH and Macůrek L: Checkpoint control and cancer. Oncogene 31: 2601-2613, 2012.

19. Li F, Huang J, Ji D, Meng Q, Wang C, Chen S, Wang X, Zhu Z, Jiang C, Shi Y, et al: Utility of urinary circulating tumor DNA for EGFR mutation detection in different stages of non-small cell lung cancer patients. Clin Transl Oncol 19: 1283-1291, 2017.

20. Xu Q, Li L, Han C, Wei L, Kong L and Lin F: Sigma-1 receptor $(\sigma 1 R)$ is downregulated in hepatic malignant tumors and regulates HepG2 cell proliferation, migration and apoptosis. Oncol Rep 39: 1405-1413, 2018.

21. Yuan X, Sun X, Shi X, Jiang C, Yu D, Zhang W, Guan W, Zhou J, Wu Y, Qiu Y and Ding Y: USP39 promotes the growth of human hepatocellular carcinoma in vitro and in vivo. Oncol Rep 34: 823-832, 2015.

22. Nagappan A, Lee WS, Yun JW, Lu JN, Chang SH, Jeong JH, Kim GS, Jung JM and Hong SC: Tetraarsenic hexoxide induces G2/M arrest, apoptosis, and autophagy via PI3K/Akt suppression and p38 MAPK activation in SW620 human colon cancer cells. PLoS One 12: e0174591, 2017.

23. Sun XT, Yuan XW, Zhu HT, Deng ZM, Yu DC, Zhou X and Ding YT: Endothelial precursor cells promote angiogenesis in hepatocellular carcinoma. World J Gastroenterol 18: 4925-4933, 2012. 
24. Hegele A, Kamburov A, Grossmann A, Sourlis C, Wowro S Weimann M, Will CL, Pena V, Lührmann R and Stelzl U: Dynamic protein-protein interaction wiring of the human spliceosome. Mol Cell 45: 567-580, 2012.

25. Tang LJ, Li Y, Liu YL, Wang JM, Liu DW and Tian QB: USP12 regulates cell cycle progression by involving c-Myc, cyclin D2 and BMI-1. Gene 578: 92-99, 2016.

26. Bulavin DV, Higashimoto Y, Popoff IJ, Gaarde WA, Basrur V, Potapova O, Appella E and Fornace AJ Jr: Initiation of a G2/M checkpoint after ultraviolet radiation requires p38 kinase. Nature 411: 102-107, 2001.

27. Tarn C, Zou L, Hullinger RL and Andrisani OM: Hepatitis B virus $\mathrm{X}$ protein activates the $\mathrm{p} 38$ mitogen-activated protein kinase pathway in dedifferentiated hepatocytes. J Virol 76: 9763-9772, 2002.
28. Huang Q, Liu X, Wu Y, Liao Y, Huang Y, Wei X and Ma M: P38 MAPK pathway mediates cognitive damage in pentylenetetrazole-induced epilepsy via apoptosis cascade. Epilepsy Res 133: 89-92, 2017.

29. Zhu G, Qiu W, Li Y, Zhao C, He F, Zhou M, Wang L, Zhao D, Lu Y, Zhang J, et al: Sublytic C5b-9 induces glomerular mesangial cell apoptosis through the cascade pathway of MEKK2-p38 MAPK-IRF-1-TRADD-caspase 8 in rat Thy-1 nephritis. J Immunol 198: 1104-1118, 2017.

30. Huang HL, Hsieh MJ, Chien MH, Chen HY, Yang SF and Hsiao PC: Glabridin mediate caspases activation and induces apoptosis through JNK1/2 and p38 MAPK pathway in human promyelocytic leukemia cells. PLoS One 9: e98943, 2014. 